\title{
Endoscopic endonasal transsphenoidal surgery: experience with 50 patients
}

\section{Hae-Dong Jho, M.D., Ph.D., and Ricardo L. Carrau, M.D.}

Departments of Neurological Surgery and Otolaryngology, University of Pittsburgh School of Medicine, Pittsburgh, Pennsylvania

An endoscope was used in transsphenoidal surgery and eventually replaced the operating microscope as a tool for visualization. Initially four patients underwent operation via a sublabial transseptal approach using a rigid endoscope in conjunction with an operating microscope. The 48 subsequent operations were performed through a nostril using only rigid endoscopes. Forty-four patients had pituitary adenomas and six had various other lesions. Thirteen patients had microadenomas, 16 had intrasellar macroadenomas, nine had macroadenomas with suprasellar extension, and six had invasive macroadenomas involving the cavernous sinus. Among eight patients with Cushing's disease, seven were cured. Of 17 patients with prolactinomas, 10 were cured clinically and chemically. Among 19 patients with nonsecreting adenomas, 16 underwent total resection and three subtotal resection, with residual tumor in the cavernous sinus. Postoperatively all patients who had undergone endonasal endoscopic surgery had unobstructed nasal airways with minimal discomfort. More than half of the patients required only an overnight hospitalization.

Key Words * chordoma * craniopharyngioma * Cushing's disease * endoscopy * pituitary neoplasms * prolactinoma * transsphenoidal approach

Although the microsurgical transsphenoidal approach has been established as the standard procedure for the surgical treatment of pituitary adenomas for decades, $[1,6,7,8]$ continuous efforts to improve these techniques and their outcome are still being made.[3-5,9-11,16,17,19-21] As an alternative to sublabial or septal incisions for transsphenoidal pituitary surgery, an endonasal microscopic technique has been reported.[3,5] Otolaryngologists have used endoscopic techniques for the treatment of most inflammatory sinonasal disorders. [18] Eventually, endoscopic sinus surgery replaced the conventional "open" method of sinus surgery. Encouraged by the increased visualization and positive results provided by endoscopy for sinonasal surgery, we have applied endoscopic techniques to transsphenoidal surgery. Initially, we used the endoscope in conjunction with an operating microscope while executing the conventional sublabial transseptal approach. As we gained confidence using the endoscope as a visualization tool, we developed an endonasal endoscopic transsphenoidal technique that did not require the use of a nasal speculum or transsphenoidal retractor. Our technique has continued to evolve. Although further technical refinement and instrument modifications are required, the short-term surgical results have been satisfactory and have provided for quick patient recovery and minimum morbidity. 
This report represents our early experience with endoscopic transsphenoidal surgery in 50 patients.

\section{CLINICAL MATERIAL AND METHODS}

\section{Patient Criteria}

Between September 1993 and June 1996, 50 patients with pituitary adenomas and several other lesions underwent operation via endoscopic techniques at the University of Pittsburgh Medical Center. Our patient population included 22 men and 28 women, ranging in age from 14 to 88 years (median 38 years). The histological diagnoses included 44 pituitary adenomas, one craniopharyngioma, one clival chordoma, one germinoma, one Rathke's cleft cyst, one postcraniotomy cerebrospinal fluid (CSF) fistula, and one metastatic adenocarcinoma.

Among the 44 patients with pituitary adenomas, 13 patients had microadenomas, 16 had intrasellar macroadenomas, nine had macroadenomas with suprasellar extension, and six had invasive macroadenomas involving the cavernous sinus. Seven patients had recurrent pituitary adenomas. Twenty-five patients had hormone-secreting pituitary adenomas (eight with Cushing's disease, 17 prolactinomas).

All of the patients with prolactinomas had received bromocriptine therapy previously. Sixteen could not tolerate bromocriptine. Another patient developed progressive pituitary dysfunction despite a good response to bromocriptine. One of the 17 prolactinomas was recurrent. Eight patients with Cushing's disease had microadenomas. One of the eight had recurrent Cushing's disease.

Among 19 patients with nonsecreting pituitary adenomas, 11 patients presented with a visual disorder, five with symptoms of pituitary apoplexy, one with progressive growth of the tumor, and two with severe intractable frontal and retroorbital headaches. Five of the nonsecreting adenomas were recurrent. Four patients in this group had previously undergone a single transsphenoidal operation and one patient had undergone transsphenoidal surgery twice, followed by fractionated conventional radiation treatment.

\section{Perioperative Evaluation and Management}

Formal postoperative endocrine evaluations were performed on all patients except for one, who was selected to be empirically treated with hormonal replacement because of her advanced age. Preoperative and postoperative magnetic resonance (MR) imaging was obtained in every patient, and CT scans were obtained preoperatively in most patients. The exquisite definition of the bony boundaries of the sinus, provided by thin-sliced axial and coronal computerized tomography (CT) scans, was essential to assess the symmetry and aeration of the sphenoid sinus and to decipher the relationship of the sphenoid sinus septum to the sella turcica floor and carotid canals.

\section{Operative Technique}

A detailed description of our technique has been reported elsewhere.[11] Our transsphenoidal operation uses an endonasal technique but without the use of a transsphenoidal retractor or nasal speculum (Fig. 1 upper). The operation is performed after general anesthesia has been induced in the patient via orotracheal intubation. The patient is maintained supine, with the head tilted to the left and the torso elevated gently. The periumbilical abdomen is also prepared for the harvest of a free fat graft. The patient, C-arm fluoroscopic device, and the endoscopic/video camera equipment are then draped following aseptic techniques. 


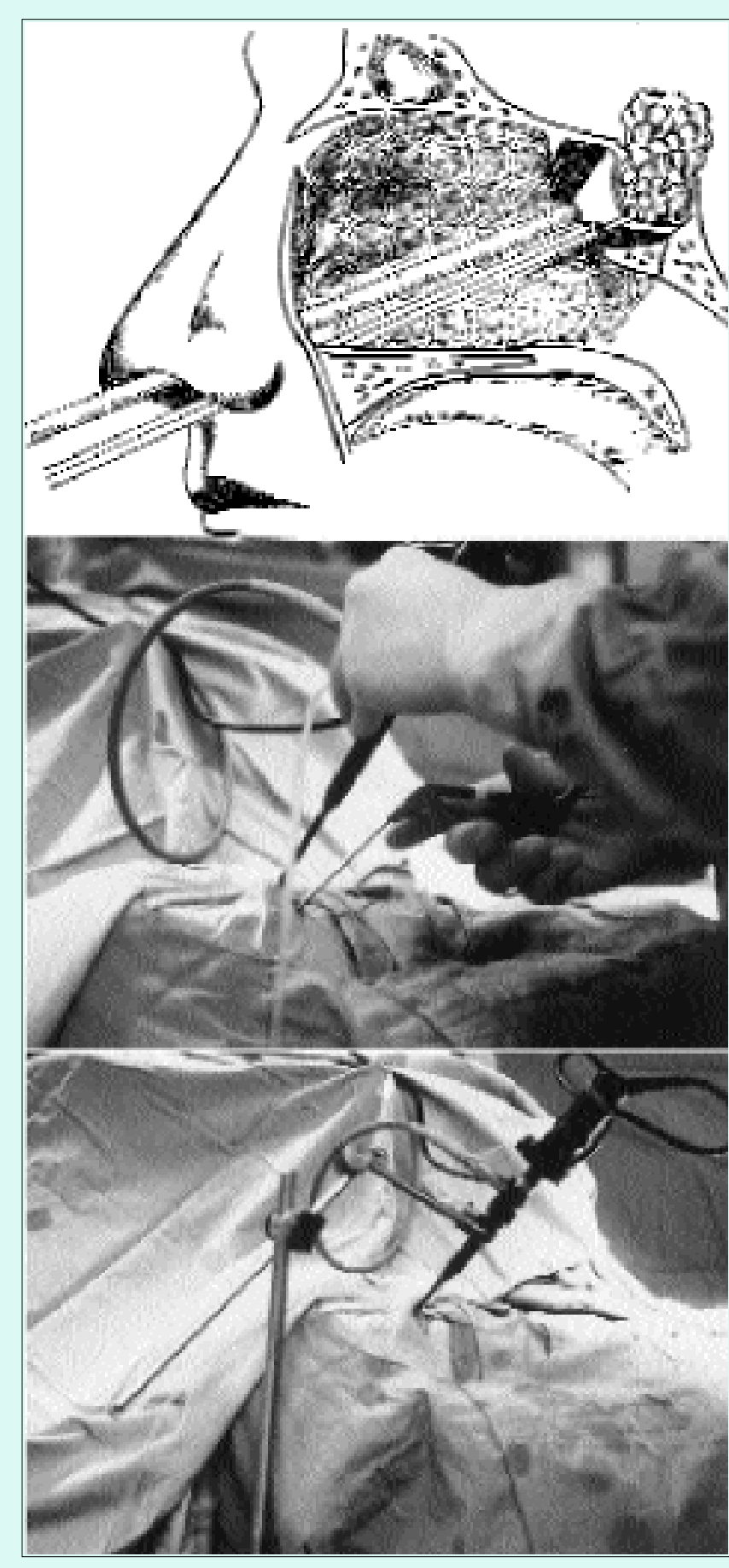

Fig. 1. Upper: Schematic drawing showing an endonasal endoscopic approach to the sella. No septal or alar incision is required. No speculum or retractor is used. Center: An endoscope is held in the surgeon's nondominant hand until an anterior sphenoidotomy is performed. Lower: Once the anterior sphenoidotomy is completed, the endoscope is mounted to an endoscope holder. This will provide the surgeon with a steady video image in addition to freeing two hands to maneuver surgical instruments simultaneously.

The first four patients in this series were treated via a sublabial transseptal transsphenoidal approach using the operating microscope as the primary instrument for tumor visualization. The endoscope was then used in addition to the operating microscope. Encouraged by these experiences, the next 48 operations were performed through the nasal cavity using only endoscopes. Only one nostril was used in most patients.

We used 4-mm rigid endoscopes with $0^{\circ}$ and $30^{\circ}$ angled lenses for 46 operations. Our endoscopes do not have a built-in work channel. Surgical instruments are inserted adjacent to the endoscope through the same nostril. The endoscope is held in the surgeon's nondominant hand until an anterior sphenoidotomy is performed (Fig. 1 center). Once the anterior sphenoidotomy is completed, the endoscope is mounted on a custom-made endoscope holder (Fig. 1 lower). This provides the surgeon with a steady video image and frees both hands to maneuver the surgical instruments. In two operations, a 5-mm-diameter stereoscopic endoscope was used. The depth perception provided by the stereoscopic view has proven to be advantageous for tumor removal. A lens cleansing irrigation-suction system has also been used. The irrigation and suction device cleans the lens of the endoscope using an irrigation system that is controlled with a pedal by the surgeon. This device virtually eliminates the need for removing the endoscope from the surgical field to clean or defog the lens.

The nasal mucosa is decongested by local application of a decongestant solution and an injection of lidocaine containing epinephrine. The middle turbinate is then outfractured to access the sphenoethmoidal recess and to identify the sphenoid sinus ostium. The sphenoid ostium is enlarged using Kerrison rongeurs. A 1.5 to $2 \mathrm{~cm}$ anterior sphenoidotomy is performed. The sphenoid septum is removed. Pieces of bone are saved for reconstruction of the anterior wall of the sella turcica. The $0^{\circ}$ endoscope provides a panoramic view of the sphenoid sinus that facilitates the identification of the optic and carotid protuberances, caroticooptic recesses, and clival indentation, in addition to the anterior wall of the sella. The $30^{\circ}$ endoscope visualizes the rostral structures better than the $0^{\circ}$ endoscope; however, 
the majority of the operation is performed using a $0^{\circ}$ endoscope, which facilitates anatomical orientation. The endoscope is now mounted on the endoscope holder.
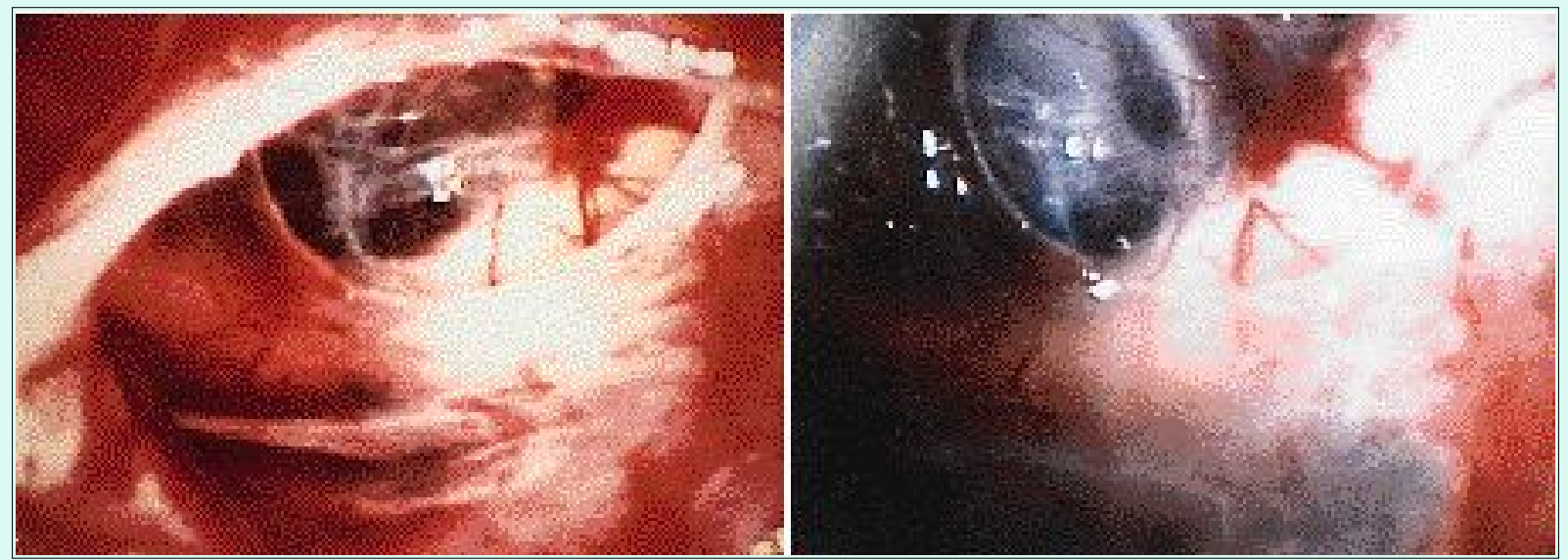

Fig. 2. Endoscopic view through the $30^{\circ}$ rigid endoscope after resection of a recurrent pituitary macroadenoma demonstrating the optic nerve and suprasellar arachnoid membrane. The anterior cerebral arteries can be seen behind the arachnoidal membrane.

The anterior wall of the sella is identified and opened using a high-speed microdrill or curettes. The dura mater is opened in cruciate fashion using curved single-blade microscissors. Under direct endoscopic visualization, the tumor is removed using micropituitary rongeurs, microsuction cannulas, and pituitary curettes while preserving the normal pituitary gland tissue. The removal of a macroadenoma requires the insertion of a 30-degree endoscope into the sella that will directly visualize suprasellar anatomy (Figs. 2 and 3).

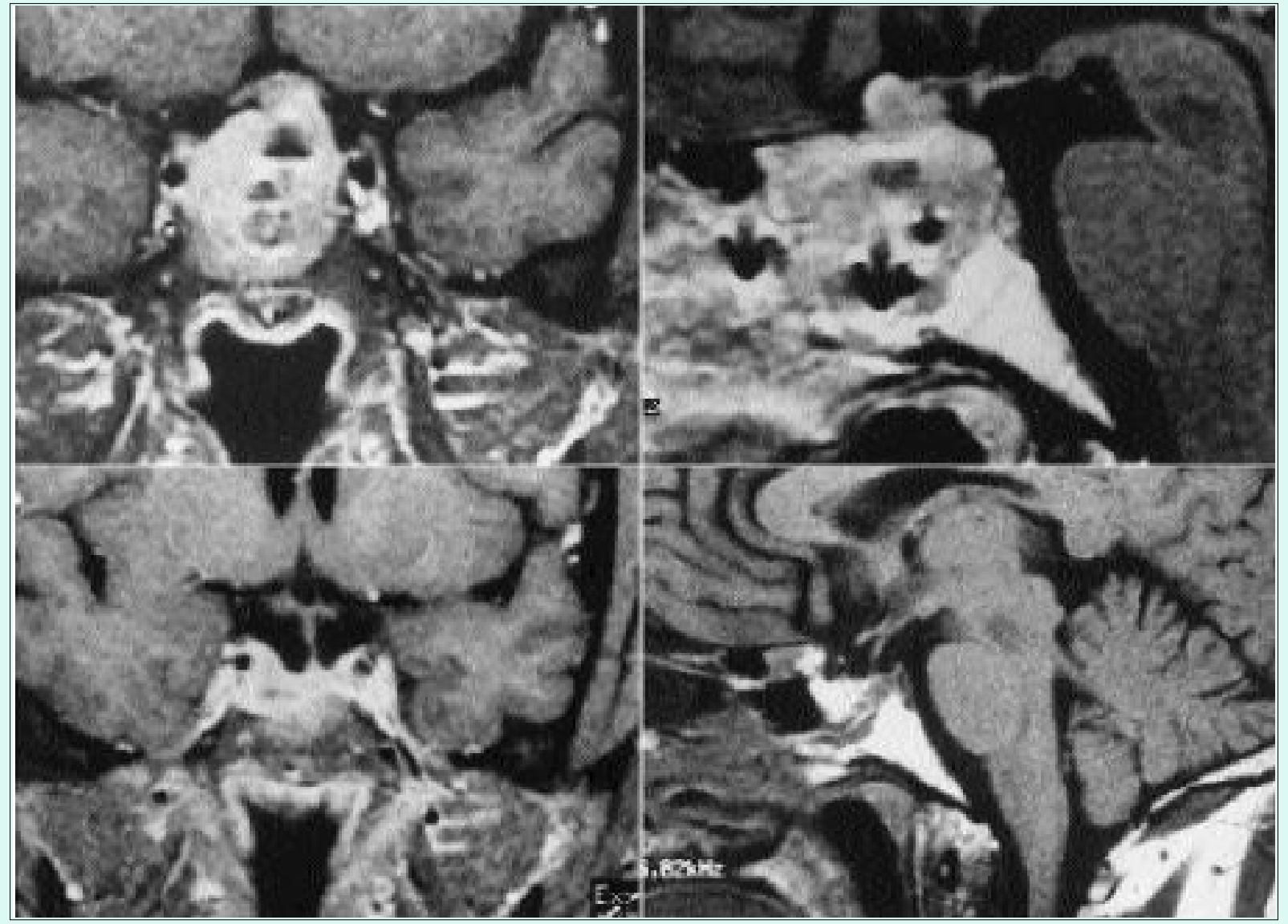

Fig. 3. Preoperative magnetic resonance images, coronal (upper left) and sagittal (upper 
right), demonstrating a recurrent pituitary macroadenoma. Postoperative magnetic resonance images, coronal (lower left) and sagittal (lower right), showing complete resection of the tumor and decompression of the optic system.

We try to preserve the arachnoidal plane, if possible. When the main tumor mass has been removed, subsequent delicate tumor resection is performed at the tumor and pituitary interface using a microdissection technique. After complete removal of the tumor, a 2-cm curvilinear skin incision is made around the inferior margin of the umbilicus to harvest a free fat graft. The free fat graft is laid in the tumor resection cavity only when the resection cavity is large or if a CSF leak is encountered intraoperatively. The anterior wall of the sella is reconstructed using the previously secured pieces of bone. When bone reconstruction is incomplete, the sphenoid sinus is then packed with an absorbable gelatin sponge to provide additional support to the fat graft. If the middle meatus is traumatized during the procedure, an absorbable gelatin film roll is used to prevent possible adhesions near the ostium of the maxillary sinus. Obstructive nasal packing is not used.

\section{RESULTS}

Postoperatively all patients who had undergone the endonasal endoscopic operation recovered with their nasal airways normal and unobstructed. Postoperative pain was reported to be minimal and the patients often did not require analgesic medication. Due to the potential occurrence of diabetes insipidus, every patient was kept in the hospital at least overnight. Among the 48 endonasal endoscopic procedures performed, 27 operations were completed with the patients needing to stay only overnight in the hospital. Eight procedures were accomplished with the patients requiring a 2-night hospitalization, and eight surgeries were completed with the patients staying for 3 nights in the hospital, primarily to observe them for the onset of diabetes insipidus. Among the 16 procedures that required the patients to remain hospitalized for 2 to 3 days postoperatively, two patients developed transient diabetes insipidus and one patient, who had a craniopharyngioma, developed permanent diabetes insipidus. The remaining 13 patients had increased postoperative urine output and were without diabetes insipidus. There were four patients who required a 4- to 5-day hospitalization due to social reasons or CSF leakage. One patient with a metastatic adenocarcinoma had a protracted hospitalization due to postoperative CSF leakage and the need for subsequent radiation treatment.

Among the eight patients whom we treated for Cushing's disease, six who underwent single operations had complete resolution of their symptoms and exhibited normalized cortisol levels postoperatively. Their other endocrine functions were normal. The other two patients required reoperation for incomplete reduction of cortisol levels after the first operation. One patient then experienced complete resolution of symptoms with postoperative panhypopituitarism. The other continued to have mildly increased cortisol levels and was eventually treated by stereotactic gamma knife surgery.

Among the 17 patients with prolactinomas, 10 patients were cured clinically and chemically, four were improved clinically with mildly elevated serum prolactin levels, and three had residual tumors in the cavernous sinuses. Two of the patients with residual prolactinomas in the cavernous sinuses were treated with gamma knife surgery, and the third patient was treated with a course of bromocriptine therapy.

Among the 19 patients with nonsecreting adenomas, 16 underwent total resection and three underwent subtotal resection because of residual tumor in the cavernous sinus. One patient with residual tumor was treated by gamma knife surgery and another with conventional fractionated radiation treatments; the third patient was selected for observation only because of his advanced age. Of the 19 patients with 
nonsecreting macroadenomas, 11 presented with progressive visual impairments. All 11 exhibited improvement of their vision and visual fields following surgery. Five patients who presented with pituitary apoplexy experienced resolution of their symptoms. Resolution of symptoms was also experienced by two patients who had severe intractable frontal and retroorbital headaches. Among this group of 19 patients, nine had anterior hypopituitarism preoperatively and three of these had improved pituitary function postoperatively. Three patients who had normal anterior pituitary functioning preoperatively developed hypopituitarism postoperatively.

One patient with a craniopharyngioma in the sellar and suprasellar region underwent total resection of the tumor. The tumor was resected with distal stalk sectioning. Rapid improvement of vision was noted postoperatively. One patient who had a sellar and suprasellar germinoma underwent subtotal resection of the tumor followed by postoperative conventional fractionated radiation treatment. This patient had improvement of diplopia postoperatively and continued to exhibit anterior hypopituitarism after surgery, as demonstrated preoperatively. One patient with a large clival chordoma underwent subtotal resection of the tumor followed by postoperative gamma knife surgery. He had undergone ventriculoperitoneal shunt placement for obstructive hydrocephalus and received fractionated conventional radiation treatment a few months earlier. We were also able to repair a postcraniotomy CSF fistula successfully by placing an abdominal free fat graft in a patient who had undergone a subfrontal resection of a craniopharyngioma previously. Another patient with a Rathke's cleft cyst was treated by successful resection of the cyst.

Two patients developed postoperative CSF leakage. The patient with metastatic adenocarcinoma developed a postoperative CSF leak after an endonasal endoscopic biopsy of the tumor. Another patient who had previously undergone transsphenoidal surgery also developed a postoperative CSF leak. The postoperative CSF leakage was successfully repaired endoscopically by using a larger abdominal free fat graft. One patient developed an asymptomatic synechia between the inferior turbinate and the nasal septum. This synechia was found during a routine postoperative follow-up visit and did not require treatment. Another patient experienced chronic sphenoid sinusitis manifesting as postpharyngeal drainage at 6 weeks postoperatively. The infection resolved after administration of antibiotic medication. No other sinunasal complications were encountered.

\section{DISCUSSION}

Microsurgical transsphenoidal surgery for pituitary adenoma has been the standard treatment for decades in the neurosurgical community.[1,2,6-8,22] Among the different techniques for transsphenoidal pituitary surgery, the sublabial transseptal approach and transnasal transseptal approach are used most commonly.[1,6-8,15,20] Although Griffith and Veerapen reported an endonasal approach to the sella in $1987,[5]$ the transsphenoidal approach via the endonasal route has not gained popularity. In 1994 Cooke and Jones [3] reported their experiences in implementing an endonasal microscopic approach to the sella with no incidence of nasal, septal, dental, or sinus complications. Our approach bears similarities to theirs as an endonasal approach; however, we do not use a nasal speculum or retractor.

Since the endoscope became popular in paranasal sinus surgery[18] and has been recently adopted in other neurosurgical procedures, interest has increased in its possible use in transsphenoidal pituitary surgery.[4,9-13,16,17,21] Papay, et al.,[13] reported a case of transseptal endoscopic repair of CSF leakage that occurred after transcranial pituitary surgery. Jankowski, et al.,[10] first reported the successful endonasal endoscopic resection of pituitary adenomas in three patients. They removed the middle turbinate to gain access to the sphenoid sinus. They performed the operation via one nostril in two 
patients and via both nostrils in another. They did not use intraoperative fluoroscopy. In their third case, they encountered technical difficulties due to septal hypertrophy in the sphenoid sinus and the hypertrophic hemorrhagic nature of the nasal mucosa. Despite the temptation to switch from the endonasal endoscopic technique to the traditional transseptal microscopic technique, they successfully finished the case with the endoscopic technique using both nostrils. Using our technique, we do not resect the middle turbinate; we simply shrink the nasal mucosa with the local application of a vasoconstrictor and we enlarge the ostium of the sphenoid sinus by an anterior sphenoidotomy. Shikani and Kelly [17] reported one case of an endoscopic biopsy of a pituitary tumor. Gamea, et al.,[4] reported their experiences with sublabial transseptal transsphenoidal pituitary surgery using an operating microscope and an endoscope. In their report of 10 patients with pituitary tumors, they claimed that the rigid endoscope facilitated better dissection of the tumor away from the normal pituitary gland. Wurster and Smith [21] briefly reported their experience using endoscopic pituitary surgery in two patients. Sethi and Pillay [16] reported approximately 40 patients in whom they had performed endoscopic pituitary surgery via either the transnasal transseptal or ethmoidectomy approach with a sphenoid retractor. Our techn ique differs from theirs in that we use an endonasal route rather than transseptal and we do not use a retractor or speculum. Our endonasal technique eliminates the use of postoperative obstructive nasal packing.

In most cases, the operation can be done through one nostril. In our series of 48 operations in 46 patients, only two operations required a two-nostril technique because of a very narrow nasal airway. The use of thin-sliced axial and coronal CT scans is essential to avoid unexpected findings from anatomical variations in the sphenoid sinus. Magnetic resonance imaging alone will not provide the necessary detail of bone anatomy of the sphenoid sinus. The decision about which nostril to use is made on the basis of the anatomy of the nasal cavity and tumor location. The approach is made through the nasal cavity that possesses the large space. Because the endonasal approach is a few degrees off midline, lateralized microadenomas are approached from the opposite nostril whenever possible.

Postoperative CSF leakage can be a major complication in transsphenoidal surgery.[2,22] In our series, abdominal fat grafts were placed in the sella when the tumor resection cavity was large or when an intraoperative CSF leak was encountered. Bone reconstruction was performed at the anterior wall of the sella, if possible. In cases of microadenoma, a fat graft was not placed.

The visualization advantage of an endoscope, especially the $30^{\circ}$ endoscope, over an operating microscope was obvious in the suprasellar region; it eliminated the need to blindly curette for a suprasellar tumor. Surgeons who favor the conventional microscopic technique may use an operating microscope and an endoscope. When a transsphenoidal speculum is used, however, the surgeon's operating space is limited by the tubular-shaped tunnel. A surgeon's mobility is restricted while using surgical instruments through this tubular space under an endoscope. The endonasal technique without the use of a retractor provides the surgeon a larger operating space, permitting the freer maneuvering of surgical instruments in the nasal cavity. Another advantage of an endoscope is that it provides a panoramic view of the sphenoid sinus so that the surgeon can recognize the bony structures covering the carotid arteries and optic nerves.[14] Although the incidence of injury to the hypothalamus, the carotid artery, and the optic system is low in the conventional microscopic technique,[2,22] direct endoscopic visualization of those structures may further reduce the risk of injury. The disadvantage of an endoscopic technique compared to the conventional microscopic surgery is that a surgeon has to operate under a two-dimensional view. Endoscopic images are still less clear and less sharp than the three-dimensional direct microscopic view. Certainly, a learning curve exists for a surgeon who is not familiar with the 
endoscope. Its use requires different skills from those necessary in microsurgical techniques because the endoscope must be handled with the nondominant hand and the surgical instruments with the other. The endoscope and surgical instruments have to enter parallel to each other in a single nostril. A surgeon inexperienced with this technique may become frustrated if the two instruments consistently strike each other in the small operating space. Practice will overcome this problem. The endoscope-holding device enables th e surgeon to use both hands and simultaneously provides a continuous view on the monitoring screen.

Future applications of the endoscope in neurosurgical practice may be enhanced by computer-guided stereotactic technology, stereoscopic endoscopes, and the development of high-definition television monitors. Computer-assisted stereotactic technology and the three-dimensional stereoscopic endoscope have already been made available. A high-definition television system may further improve clarity and sharpness of images. The technique of endonasal endoscopic pituitary surgery can be simplified and refined and may benefit from the modification of surgical instruments.

\section{Acknowledgment}

The authors wish to thank Arthur P. Nestler, B.S.N., and Robin A. Coret, B.A. for their assistance in preparation of this manuscript.

\section{References}

1. Adams CBT, Burke CW: Current modes of treatment of pituitary tumors. Br J Neurosurg 7:123-127, 1993

2. Black PMcL, Zervas NT, Candia G: Incidence and management of complications of transsphenoidal operation for pituitary adenomas. Neurosurgery 20:920-924, 1987

3. Cooke RS, Jones RAC: Experience with the direct transnasal transsphenoidal approach to the pituitary fossa. Br J Neurosurg 8:193-196, 1994

4. Gamea A, Fathi M, el-Guindy A: The use of the rigid endoscope in trans-sphenoidal pituitary surgery. J Laryngol Otol 108:19-22, 1994

5. Griffith HB, Veerapen R: A direct transnasal approach to the sphenoid sinus. Technical note. J Neurosurg 66:140-142, 1987

6. Guiot A: Transsphenoidal approach in surgical treatment of pituitary adenomas: General principles and indications in nonfunctioning adenoma, in Kohler PO, Ross GT (eds): Diagnosis and Treatment of Pituitary Tumors. Amsterdam: Excerpta Medica, 1973, pp 159-178

7. Hardy J: Trans-sphenoidal approach to the pituitary gland, in Wilkins RH, Rengachary SS (eds): Neurosurgery. New York: McGraw-Hill, 1985, Vol 1, pp 889-898

8. Hardy J: Transphenoidal microsurgery of the normal and pathological pituitary. Clin Neurosurg 16:185-217, 1969

9. Helal MZ: Combined micro-endoscopic trans-sphenoid excisions of pituitary macroadenomas. Eur Arch Otorhinolaryngol 252:186-189, 1995 
10. Jankowski R, Auque J, Simon C, et al.: Endoscopic pituitary tumor surgery. Laryngoscope 102:198-202, 1992

11. Jho HD, Carrau RL, Ko Y: Endoscopic pituitary surgery, in Wilkins RH, Rengachary SS (eds): Neurosurgical Operative Atlas. Park Ridge, Ill: American Association of Neurological Surgeons, 1996, Vol 5, pp 1-12

12. Liston SL, Siegel LG, Thienprasit P, et al: Nasal endoscopes in hypophysectomy. J Neurosurg 66:155, 1987 (Letter)

13. Papay FA, Benninger MS, Levine HL, et al: Transnasal transseptal endoscopic repair of sphenoidal cerebral spinal fluid fistula. Otolaryngol Head Neck Surg 101:595-597, 1989

14. Renn WH, Rhoton AL Jr: Microsurgical anatomy of the sellar region. J Neurosurg 43:288-298, 1975

15. Rosegay H: Cushing's legacy to transsphenoidal surgery. J Neurosurg 54:448-454, 1981

16. Sethi DS, Pillay PK: Endoscopic management of lesions of the sella turcica. J Laryngol Otol 109:956-962, 1995

17. Shikani AH, Kelly JH: Endoscopic debulking of a pituitary tumor. Am J Otolaryngol 14:254-256, 1993

18. Stammberger H: Endoscopic endonasal surgery--concepts in treatment of recurring rhinosinusitis. Part II. Surgical technique. Otolaryngol Head Neck Surg 94:147-156, 1986

19. Stevens MH, Apfelbaum RI: Transnasal pituitary tumor surgery. Laryngoscope 100:427-429, 1990

20. Wilson WR, Khan A, Laws ER Jr: Transseptal approaches for pituitary surgery. Laryngoscope 100:817-819, 1990

21. Wurster CF, Smith DE: The endoscopic approach to the pituitary gland. Arch Otolaryngol Head Neck Surg 120:674, 1994 (Letter)

22. Zervas NT: Surgical results in pituitary adenomas: results of international survey, in Black PM, Zervas NT, Ridway EC Jr, et al (eds): Secretory Tumors of the Pituitary Gland. New York: Raven Press, 1984, pp 377-385

Manuscript received June 24, 1996.

Accepted in final form June 28, 1996.

Address reprint requests to: Hae-Dong Jho, M.D., Ph.D., Department of Neurological Surgery, Presbyterian University Hospital, Suite B-400, 200 Lothrop Street, Pittsburgh, Pennsylvania 15213. 